\title{
1 Sperm head abnormalities are more frequent in songbirds with more 2 helical sperm: A possible trade-off in sperm evolution
}

\section{Abstract}

Sperm morphology varies enormously across the animal kingdom. While knowledge of the factors that drive the evolution of interspecific variation in sperm morphology is accumulating, we currently have little understanding of factors that may constrain evolutionary change in sperm traits. We investigated whether susceptibility to sperm abnormalities could represent such a constraint in songbirds, a group characterised by a distinctive helical sperm head shape. Specifically, using 36 songbird species and data from light- and scanning electron microscopy, we examined amongspecies correlations between the occurrence of sperm head abnormalities and sperm morphology, as well as the correlation between sperm head abnormalities and sperm competition (estimated as the frequency of extra-pair young). We found that species with more helically shaped sperm heads (i.e. a wider helical membrane and more pronounced cell waveform) had a higher percentage of abnormal sperm heads than species with relatively straight sperm heads, and that sperm head traits were better predictors of head abnormalities than total sperm length. In contrast, there was no correlation between sperm abnormalities and the frequency of extra-pair young. Given that songbird species with more pronounced helical sperm have higher average sperm swimming speed, our results suggest an evolutionary trade-off between sperm performance and the structural integrity of the sperm head. As such, susceptibility to morphological abnormalities may constrain the evolution of helical sperm morphology in songbirds. We recommend further studies into such evolutionary constraints, as well as investigating the relationship between sperm morphology and sperm integrity in other taxa with helical sperm morphology.

Keywords: acrosome - cell integrity - passerine bird - sperm competition - sperm damage - sperm morphology 


\section{Introduction}

Over the last few decades, much attention has been paid to understanding factors that drive the evolutionary diversification of sperm morphology. For example, it is now well established that variation in sperm length is related to the risk of sperm competition or the size of female sperm storage organs in a wide range of taxa (Miller \& Pitnick, 2002; Pitnick et al. 2009). In contrast, research into factors that may constrain evolutionary change in sperm morphology is generally lacking. One potentially limiting factor is the need for males to consistently produce morphologically normal sperm; as normal sperm are critical to successful fertilization and thus male fitness. Indeed, a decrease in the frequency of morphologically normal sperm is associated with decreased fertility in humans (Nikolettos et al., 1999), domestic poultry (Wishart et al., 1986), and deer (Malo et al., 2005). Thus, whilst selection might drive the evolution of sperm traits that optimise performance (e.g. increase swimming speed), such traits might not readily evolve if they are associated with an increased risk of morphological abnormalities.

Sperm abnormalities can be caused by a number of factors, including heat stress (Hurley et al., 2018), mechanical damage (e.g. centrifugation, Katkov \& Mazur, 1998), and oxidative stress (Aitken \& Baker, 2006). Due to their high polyunsaturated fatty acid (PUFA) content, sperm cells are especially vulnerable to oxidation-induced damage (Aitken \& Baker, 2006; Constantini et al., 2010), and oxidative stress has been shown to result in sperm abnormalities and decreases in sperm fertilizing capacity (e.g. Hsu et al., 1999; reviewed in Costantini et al. 2010). Although oxidative stress is an ongoing challenge for all sperm, susceptibility of sperm to oxidative stress can be influenced by environmental factors, e.g. exacerbated at high levels of radioactivity (Møller et al., 2005) or alleviated with dietary antioxidants (Tomášek et al., 2017). Hermosell et al. (2013) showed that sperm head abnormalities in 10 songbird species were significantly higher in radioactive Chernobyl areas than in unpolluted areas, demonstrating that sperm abnormalities occur in wild populations at 
variable rates. Thus, the capacity of sperm to avoid damage may be especially important for males in populations exposed to environmental stressors such as radioactivity.

Similarly, in populations with intense sperm competition, high rates of abnormal sperm could be associated with particularly high fitness costs. As such, sperm competition may select for reductions in the occurrence of sperm abnormalities by favouring mechanisms that protect sperm from oxidative damage and/or minimize sperm production errors during spermatogenesis. The proportion of abnormal sperm is negatively correlated with the level of sperm competition in both mammals (rodents, Montoto et al., 2011) and birds (fairy-wrens, Rowe \& Pruett-Jones, 2011). However, it is unclear whether sperm shape and structure play functional roles in these processes; for example whether populations with higher levels of sperm competition evolve more structurally robust sperm.

In songbirds (Passeriformes: Passerida), abnormalities of the sperm head are found more frequently in species that have longer sperm (Hermosell et al., 2013), indicating a link between sperm size and sperm head integrity. However, it is unclear why longer sperm should be particularly susceptible to damage in the head region. Songbirds have a characteristic helical sperm head, the extent of which varies among species (Jamieson, 2007, Støstad et al., 2018), often with a wide helical membrane spiralling around the helical acrosome (Jamieson, 2007). The extent of this helical shape is associated with total sperm length, such that longer sperm have a more pronounced helical form and a larger helical membrane (Støstad et al., 2018). Given this, we hypothesised that the helical shape of sperm heads could be driving the relationship between sperm size and the occurrence of sperm head abnormalities across species. We therefore investigated the relationship between sperm head abnormalities and sperm morphology (i.e. total sperm length and sperm head morphology) using data from light- and scanning electron microscopy (SEM) for 36 songbird species. In addition, we used a subset of these species $(n=32)$ to examine the association between the frequency of 
abnormal sperm head morphology and the level of sperm competition (estimated as the rate of extra-pair young).

\section{Methods}

We used light microscopy to measure total sperm length and score the occurrence of sperm head abnormalities, whereas sperm head morphology data were taken from Støstad et al. (2018). For light microscopy, we used fixed sperm samples ( $5 \%$ formaldehyde) from the collection at the Natural History Museum in Oslo (NHMO). Ten individuals from each of the 36 species were chosen, except for the white-throated dipper (Cinclus cinclus) and the nuthatch (Sitta europaea), for which only 9 and 8 individuals were available. A small aliquot of formaldehyde-fixed sperm was smeared onto a microscope slide, air-dried, and rinsed with distilled water, before being imaged using a digital light microscope (DM6000 B, Leica Microsystems), which was set to 320x magnification and fitted with a digital camera (DFC420, Leica Microsystems, Heerbrugg, Switzerland). From these images we measured total sperm length ( $\pm 0.1 \mu \mathrm{m}, 10$ intact/morphologically normal cells per individual) using Leica Application Suite (version 2.6.0 R1).

To assess the frequency of sperm head abnormalities, we examined sperm smears using a light microscope and assessed at least 100 randomly selected sperm from each male, scoring sperm as having either a "normal head" or an "abnormal head". We were unable to assess 100 sperm in 39 cases (10.9\%); in these instances, we set a minimum threshold for assessment of 50 sperm (median of all samples: 103, range: 50-162, see Table S1). A normal head was defined as a head with no signs of damage and a shape that corresponded to the usual appearance for the species (Fig. 1a). An abnormal head was either 1) a head which appeared to have lost its acrosome, Fig. 1b); or 2) a head that appeared abnormal in shape (Fig. 1C). Of all sperm classified as "abnormal", 76\% were placed in the first category, and $24 \%$ in the second. The two categories were combined for analysis to avoid an excessive number of models, though when analysed separately the results were qualitatively similar (Table S2). We considered the idea that pipetting could be physically damaging to sperm cells. To 
investigate this, we took aliquots of samples from two males and prepared smears of these aliquots using small, medium or large pipette tip openings (i.e. a total of 3 smears per male) and assessed the frequency of sperm with abnormal head morphology for each aliquot. The different preparation techniques did not appear to impact the frequency of abnormal heads (male 1: $37 / 43 / 42 \%$, male 2: $53 / 30 / 41 \%)$.

Data on sperm head morphology (species mean values) were taken from Støstad et al. (2018). These data (Table S3) are SEM-derived length measurements, from an average of 6.6 males per species and 9.5 cells per male. Specifically, we used digital image analysis (ImageJ 1.50i) to obtain measures of sperm head morphology from SEM images for all 36 songbird species, including several one-dimensional traits of the sperm head (acrosome length, nucleus length, and helical membrane width). We also calculated sperm head volume, as well as the variable 'waveform', which is the ratio of straight line to centre line head length and gives an indication of the amplitude of the helical shape of the 'core' of the sperm head. For further details of measurement, see supplementary material (Fig. S1). Finally, we used data on extra-pair young for the 32 species given in Støstad et al. (2018) as our proxy for the level of sperm competition (Table S3).

\section{Statistical analysis}

Statistical analyses were conducted in R v 3.3.3 (R Core Team 2017). Percentages of abnormal sperm were logit transformed due to the binomial structure of the data, as was the rate of extra-pair young, whereas all sperm morphology traits were log transformed. We tested whether the percentage of abnormal sperm heads varied among species using a Kruskal-Wallis test due to heteroscedasticity. We then used mean values for each species for further analysis. To determine the relationship between sperm morphology traits and the percentage of abnormal sperm heads whilst accounting for phylogeny, we performed Phylogenetic Generalised Least Squares (PGLS) regressions using the pgls function of the caper package (Orme, 2013) and the phylogenetic tree from Støstad et al. (2018). First, we constructed seven separate PGLS models with the percentage of abnormal sperm as the 
response variable and each of the seven sperm morphology traits as the predictor variables. In a separate analysis, we found a positive correlation between age of the sample (average years between collection and microscopy) and the frequency of abnormal sperm heads (GLMM, $r=0.26, p$ $<0.001$ ), which we speculate may be related to a decrease in the $\mathrm{pH}$ of the fixative over time. To account for this, we included 'sample age' as a covariate in all analyses, though this was not significant in any model (all $p>0.1$ ). Two species showed a significant relationship between sample age and the frequency of abnormal sperm, but when we removed these from the main analyses our results were qualitatively similar, thus we present the models using all species in the main text.

Second, to determine the contribution of each of the sperm morphology traits to the percentage of abnormal sperm heads, we performed a backwards model selection procedure, starting with all sperm morphology traits as the predictors in a single PGLS model, with stepwise removal of variables with the highest $p$-value until there were no non-significant terms. For all models, variance inflation factors were below 4.5 (well under the recommended cut-off value of 10, Kleinbaum et al., 2007). The model with the lowest AICc score was considered the best model, though models with a $\triangle \mathrm{AICC} \leq 2$ were considered equally plausible (Burnham \& Anderson, 2003). Finally, we assessed the relationship between sperm competition and sperm abnormalities using a PGLS regression, with the rate of extra-pair young as the predictor and the percentage of abnormal sperm heads as the response variable.

\section{Results}

The percentage of sperm with abnormal head morphology varied from $0 \%$ to $82.5 \%$ among individuals, and species mean values ranged from $1.8 \%$ to $36 \%$ (Fig. 2; overall mean: $13.5 \%$; $\mathrm{H}_{35}=$ 134.6, $p<0.001)$. Using PGLS regression, we found that all sperm morphological traits were significantly positively correlated with the percentage of abnormal sperm (Table 1), with the exception of nucleus length for which the correlation was negative (Table 1). The strongest relationship was the correlation between the percentage of abnormal sperm and helical membrane 
width, which was stronger than the correlation between abnormal sperm and total sperm length $(r=$ 0.73 vs $r=0.39$, helical membrane width and total sperm length respectively, Fig. 3A and 3B). Next, using a PGLS model selection procedure, we found that the best fit model included helical membrane width (Fig. 3A) and waveform (Fig. 3C) as the only predictors (Table 2), although an equally plausible model also included acrosome length as a non-significant predictor (Table 2). In contrast, we found no relationship between the percentage of abnormal sperm and the rate of extra-pair young (Table 1). Finally, qualitative assessment of SEM images suggested that sperm with abnormal head morphology exhibited acrosomal membranes that had disintegrated; in particular, the helical membrane appeared to be compromised or damaged (Fig. 4).

\section{Discussion}

159 We found that sperm head abnormalities were correlated with all measured sperm morphology 160 traits, but that helical membrane width and the waveform of the sperm head 'core' were the most important predictors for explaining the proportion of abnormal sperm heads in a multivariate model. Together, membrane width and waveform describe the characteristic helical head shape of songbird sperm (Støstad et al. 2018). Thus, our results suggest that it is not simply longer sperm with a longer acrosome that are more likely to exhibit abnormal morphology (as suggested by Hermosell et al., 2013), but that species with sperm that exhibit a stronger helical head shape also show higher rates of sperm head abnormalities.

From the perspective of individual males, a high frequency of abnormal sperm is likely to negatively impact fertilisation success. Indeed, at least in domestic fowl, fertility has been shown to decline as the proportion of morphologically abnormal sperm increases (Wishart et al., 1986). Thus, if more helical sperm are particularly prone to such abnormalities, as our results suggest, there could be a fitness cost associated with producing sperm with a more pronounced helical shape. However, a helical sperm shape may also confer a potential fertilization advantage; across species, sperm with a more helical form swim faster (Støstad et al., 2018). Thus, our findings indicate a possible 
evolutionary trade-off in sperm structure and performance: more helical sperm with wider membranes swim faster, but at the cost of being more fragile. As such, susceptibility to morphological abnormalities could then be considered an evolutionary constraint, limiting the morphological diversification of helical sperm. Although our work is based on songbirds, we suggest that such a trade-off scenario may be applicable to other taxa exhibiting helical sperm morphology, e.g. sea hares (Kubo \& Ishikawa, 1981), dogfish (Stanley, 1971) and octopuses (Maestre et al., 1982), and we recommend that future studies should investigate the generality of our findings across taxa, as well as the fitness consequences of sperm head abnormalities within taxa.

We did not find an association between the proportion of sperm with abnormal head morphology and the level of sperm competition, which stands in contrast to previous studies (Montoto et al., 2011; Rowe \& Pruett-Jones, 2011). This is intriguing, as sperm numbers are a key determinant of the outcome of sperm competition in a range of taxa, including passerine birds (Parker, 1982; Birkhead \& Pizzari, 2002), and thus it is generally expected that there would be strong selection for maintaining sperm integrity (i.e. maximising the number of viable sperm, cf. Hunter \& Birkhead, 2002) in species with intense sperm competition. However, the effect of sperm competition might depend on the relative importance of sperm number versus other sperm traits (e.g. sperm size) in any given system (cf. Immler et al., 2011). Thus one potential explanation for the difference between our study and previous studies (Montoto et al., 2011; Rowe \& Pruett-Jones, 2011 ) is that the relative importance of sperm numbers for male fitness differs among the groups of taxa examined. Indeed, the study by Rowe and Pruett-Jones (2011) was taxonomically limited to the Australian Maluridae (fairy-wrens and allies), a system characterised by extremely high investment in in sperm production and in which sperm numbers are thought to be particularly important for male reproductive success (Tuttle et al., 1996, Pruett-Jones \& Tuttle 2007). In contrast, given our use of a substantially broader taxonomic range in the current study, selection pressures may be more variable across the species used, which may in turn reduce or obscure any correlation. As such, it may be that the relationship between sperm competition and the proportion of morphologically 
normal sperm is not a general pattern, but rather reflects a clade-specific response to selection for sperm quantity.

In the current study, we observed a relationship between sperm structure and the occurrence of morphologically abnormal sperm, though the mechanism underlying this pattern is unknown. Unfortunately, little research exists on the relationship between cell integrity and sperm shape, or, indeed, any cell shape. However, we suggest that one plausible mechanism involves susceptibility to oxidative stress. Sperm cells are particularly vulnerable to attack by reactive oxygen species due to the high PUFA content of sperm membranes, and such attacks can induce a lipid peroxidation chain event with consequent damage to the cell membrane (Surai et al., 2001; Aitken \& Baker, 2006). In humans, the presence of specific PUFAs is related to sperm abnormalities and male infertility (Aksoy et al., 2006). Although it is unknown whether this is also the case for birds, the relative proportions of different PUFAs as well as the total amount of PUFAs have been shown to vary among species of Galloanserae (fowl and ducks; Surai et al., 2001). If sperm PUFA content and composition varies among species of passerine birds, it may be that species with wider helical membranes also have higher levels of PUFAs in their sperm (or higher levels of specific PUFAs that are more strongly linked to oxidative damage), which could in turn explain the link between sperm morphology and susceptibility to morphological abnormalities. Our finding that the proportion of sperm with head abnormalities was positively correlated with sperm head volume offers some tentative support for this idea; a larger sperm head is likely to have a larger membrane surface area and thus potentially also a higher PUFA content. Moreover, oxidative damage to cell membranes has been shown to lead to premature acrosome reactions (Hsu et al., 1999), and in the SEM images we acquired, it appeared that the acrosomal membranes were disintegrating, which is part of the acrosome reaction process at fertilisation. Thus, our observations suggest that wide helical membranes could be more prone to premature acrosome reactions due to an increased susceptibility to oxidative stress, and we recommend future studies investigate these mechanisms further. 
An alternative explanation for the abnormal sperm we observed is that they were damaged

227 by the sampling or preservation process. Formaldehyde is effective at cross-linking proteins

228 (Thavarajah et al. 2012), but is less effective at cross-linking lipids (including PUFAs); in fact,

formaldehyde fixation can lead to PUFAs being lost from the cell membranes over time (Jones, 1969).

As such, the observed sperm abnormalities may have been due to poor fixation. However, when our findings. sperm, only some males were affected, suggesting that abnormalities might be the result of environmental factors that differentially affect individuals or populations (as emphasised by Reinhardt et al. 2015). Examples of such factors include radioactivity (Hermosell et al. 2013), disease (Rueffer et al. 2011), and diet (e.g. deficiency in dietary nutrients, Watanabe \& Endo, 1991). Most notably, the PUFA content of the diet may influence the PUFA content of sperm (Cerolini et al. 2003), affecting the risk of sperm damage through lipid peroxidation, whereas high levels of antioxidants in

243 the diet could alleviate such oxidative damage (Tomášek et al. 2017). Thus, the intraspecific variation

244 we observed may reflect variation in the diet or condition of individuals more than it reflects

245 intraspecific variation in sperm head morphology (which is relatively low compared to interspecific 246 variation, Støstad et al., 2018). Finally, we note that sperm with a more distinct helical shape could potentially be more prone to production errors during spermatogenesis than straighter sperm, as the spermatogenesis of helical sperm differs from that of other types of animal sperm (Stanley, 1971); though it has not been demonstrated whether this is the case. Importantly, such errors during spermatogenesis also do not exclude the possibility of subsequent oxidative damage to sperm, or 
252 differentiation of normal sperm and thus result in the production of sperm with abnormal

253 morphology (Constatini et al., 2010).

254 In conclusion, our results demonstrate that songbirds with more helically shaped sperm are more prone to sperm abnormalities, and that this could act as a constraint on the evolution of helical sperm morphology. The trade-off between sperm swimming speed and structural integrity provides a novel framework in which to consider the evolution of sperm in songbirds as well as other taxa with

258 similar sperm morphology, and contributes to improving our understanding of sperm evolution in a 259 broader sense.

\section{Ethics}

Sampling was conducted in adherence to ethical guidelines for use of animals in research and with permission from all relevant local authorities.

\section{Acknowledgements}

265 We thank all contributors to the sperm bank and the sperm database at NHMO. 


\section{References}

268

Aitken, R.J. \& Baker, M.A. 2006. Oxidative stress, sperm survival and fertility control. Mol. Cell. Endocrinol. 250: 66-69.

Aksoy, Y., Aksoy, H., Altınkaynak, K., Aydın, H.R. \& Özkan, A. 2006. Sperm fatty acid composition in subfertile men. Prostaglandins Leukot. Essent. Fatty Acids 75: 75-79.

Birkhead, T. R., \& Pizzari, T. 2002. Postcopulatory sexual selection. Nature Rev. Genet., 3: 262-273.

Burnham, K.P. \& Anderson, D.R. 2003. Model selection and multimodel inference: a practical information-theoretic approach. Springer Science \& Business Media.

Cerolini, S., Pizzi, F., Gliozzi, T., Maldjian, A., Zaniboni, L. \& Parodi, L. 2003. Lipid manipulation of chicken semen by dietary means and its relation to fertility: a review. Worlds Poult. Sci. J. 59: 65-75.

Costantini, D., Rowe M., Butler, M.W. \& McGraw, K.J. 2010. From molecules to living systems: historical and contemporary issues in oxidative stress and antioxidant ecology. Funct. Ecol. 24: 950-959.

Hermosell, I.G., Laskemoen, T., Rowe, M., Møller, A.P., Mousseau, T.A., Albrecht, T., et al. 2013. Patterns of sperm damage in Chernobyl passerine birds suggest a trade-off between sperm length and integrity. Biol. Lett. 9: 20130530.

Hsu, P.C., Hsu, C.C. \& Guo, Y.L. 1999. Hydrogen peroxide induces premature acrosome reaction in rat sperm and reduces their penetration of the zona pellucida. Toxicology 139: 93-101.

Hunter, F.M. \& Birkhead, T.R. 2002. Sperm viability and sperm competition in insects. Curr. Biol. 12: $121-123$.

Hurley, L.L., McDiarmid, C.S., Friesen, C.R., Griffith, S.C. \& Rowe, M. 2018. Experimental heatwaves negatively impact sperm quality in the zebra finch. Proc. R. Soc. Lond. B. Biol. Sci. 285 : 20172547. 
Immler, S., Pitnick, S., Parker, G.A., Durrant, K.L., Lüpold, S., Calhim, S., et al. 2011. Resolving variation in the reproductive tradeoff between sperm size and number. Proc. Natl. Acad. Sci. U. S. A. 108: $8065-8065$.

Jamieson, B.G.M. 2007. Avian spermatozoa: structure and phylogeny. In: Reproductive biology and phylogeny of birds (ed. Jamieson BGM). New Hampshire: Science Publishers.

Jones, D. 1969. The reaction of formaldehyde with unsaturated fatty acids during histological fixation. Histochem. J. 1: 459-491.

Katkov, I.I. \& Mazur, P. 1998. Influence of centrifugation regimes on motility, yield, and cell associations of mouse spermatozoa. J. Androl. 19: 232-241.

Kubo, M. \& Ishikawa, M. 1981. Organization of the acrosome and helical structures in sperm of the Aplysiid, Aplysia kurodai (Gastropoda, Opisthobranchia). Differentiation 20: 131-140.

Kleinbaum, D., Kupper, L., Nizam, A. \& Muller, K. 2007. Applied regression analysis and other multivariable methods. Boston: PWS-KENT Publishing Company.

Maestre, M.F., Bustamante, C., Hayes, T.L., Subirana, J.A. \& Tinoco, I. 1982. Differential scattering of circularly polarized light by the helical sperm head from the octopus Eledone cirrhosa. Nature 298: 773-774.

Malo, A.F., Garde, J.J., Soler, A.J., Garcia, A.J., Gomendio, M. \& Roldan, E.R.S. 2005. Male fertility in natural populations of red deer is determined by sperm velocity and the proportion of normal spermatozoa. Biol. Reprod. 72: 822-829.

Miller, G. T., \& Pitnick, S. 2002. Sperm-Female Coevolution in Drosophila. Science 298: 1230-1233.

Montoto, L.G., Magaña, C., Tourmente, M., Martín-Coello, J., Crespo C., Luque-Larena J.J., et al. 2011. Sperm competition, sperm numbers and sperm quality in muroid rodents. PLoS One 6: e18173.

Møller, A.P., Surai, P. \& Mousseau, T.A. 2005. Antioxidants, radiation and mutation as revealed by sperm abnormality in barn swallows from Chernobyl. Proc. R. Soc. Lond. B. Biol. Sci. 272: 247252. 
Nikolettos, N., Küpker, W., Demirel, C., Schöpper, B., Blasig, C., Sturm, R., et al. 1999. Fertilization potential of spermatozoa with abnormal morphology. Hum. Reprod. 14: 47-70.

Orme, D. 2013. The caper package: comparative analysis of phylogenetics and evolution in R. $R$ package version 5: 2 .

Parker, G. A. 1982. Why are there so many tiny sperm? Sperm competition and the maintenance of two sexes. J. Theor. Biol. 96: 281-294.

Pitnick, S., Hosken, D.J. \& Birkhead, T.R. 2009. Sperm morphological diversity. In S. Pitnick, D. J. Hosken, T. R. Birkhead, eds. Sperm biology: An evolutionary perspective. Academic Press, Cambridge, Massachusetts.

Pruett-Jones, S. \& Tuttle, E.M. 2007. Fairy-wren sperm counts: a correction. Anim. Behav. 73: e1-e2.

R Core Team. 2017. R: A language and environment for statistical computing. Vienna, Austria: $R$ Foundation for Statistical Computing. http://www.R-project.org/

Reinhardt, K., Dobler, R. \& Abbott, J. 2015. An ecology of sperm: sperm diversification by natural selection. Annu. Rev. Ecol. Evol. Syst. 46: 435-459.

Rowe, M. \& Pruett-Jones, S. 2011. Sperm competition selects for sperm quantity and quality in the Australian Maluridae. Plos One 6: e15720.

Rueffer, U., Breuer, K., Josting, A., Lathan, B., Sieber, M., Manzke, O., et al. 2001. Male gonadal dysfunction in patients with Hodgkin's disease prior to treatment. Ann. Oncol. 12: 1307-1311.

Stanley, H.P. 1971. Fine structure of spermiogenesis in the elasmobranch fish Squalus suckleyi. II. Late stages of differentiation and structure of the mature spermatozoon. J. Ultrastruct. Res. 36: 103-118.

Støstad, H.N., Johnsen, A., Lifjeld, J.T. \& Rowe, M. 2018. Sperm head morphology is associated with sperm swimming speed: A comparative study of songbirds using electron microscopy. Evolution 72: 1918-1932. 
Surai, P.F., Fujihara, N., Speake, B.K., Brillard, J.P., Wishart, G.J. \& Sparks, N.H.C. 2001. Polyunsaturated fatty acids, lipid peroxidation and antioxidant protection in avian semen. Asian-Australas. J. Anim. Sci. 14: 1024-1050.

Thavarajah, R., Mudimbaimannar, V.K., Elizabeth, J., Rao, U.K. \& Ranganathan, K. 2012. Chemical and physical basics of routine formaldehyde fixation. J. Oral. Maxillofac. Pathol. 16: 400-405.

Tomášek, O., Albrechtová, J., Němcová, M., Opatová, P. \& Albrecht, T. 2017. Trade-off between carotenoid-based sexual ornamentation and sperm resistance to oxidative challenge. Proc. $R$. Soc. Lond. B. Biol. Sci. 284: 20162444.

Tuttle, E.M., Pruett-Jones, S. \& Webster, M.S. 1996. Cloacal protuberances and extreme sperm production in Australian fairy-wrens. Proc. R. Soc. Lond. B. Biol. Sci. 263: 1359-1364.

Watanabe, T., \& Endo, A. 1991. Effects of selenium deficiency on sperm morphology and spermatocyte chromosomes in mice. Mutat. Res. Lett. 262: 93-99.

Wishart, G.J. \& Palmer, F.H. 1986. Correlation of the fertilising ability of semen from individual male fowls with sperm motility and ATP content. Br. Poult. Sci. 27: 97-102.

\section{Figure legends}

Figure 1. Light microscope images (320 x magnification) showing three categories of sperm head morphology in the American redstart (Setophaga ruticilla): A) a normal sperm head, B) a sperm head with missing acrosome, C) a misshapen sperm head. Scale bars are $10 \mu \mathrm{m}$.

Figure 2. Percentage of abnormal sperm heads in 36 songbird species, ordered according to phylogeny, i.e. species next to each other are more closely related. The bold line shows the median value, boxes show first and third quartile, whiskers show extreme data within 1.5 IQR of the lower/upper quartile. Circles represent outliers. 
364 Figure 3. Relationship between the percentage of abnormal sperm heads and A) membrane width, B)

365 total sperm length and C) waveform, for 36 songbird species. Values are not log transformed (unlike

366 in all analyses); lines are simple regression lines.

367 Figure 4. Examples of sperm heads of two types of morphology in three different states; A) a

368 morphologically normal sperm from the reed bunting (Emberiza schoeniclus), which has a wide

369 helical membrane and a pronounced waveform, and B) a partially damaged reed bunting sperm; and

370 C) an abnormal/fully damaged sperm from the chaffinch (Fringilla coelebs), which is similar to the

371 reed bunting in morphology. Images are taken with a scanning electron microscope, at

372 magnifications of $7000 \times(A, B)$ and $10000 \times(C)$. Images are not mirrored during processing. 


\section{Tables}

Table 1. PGLS analyses of sperm head abnormalities (\%) in relation to sperm traits across 36 songbird species, as well as in relation to the rate of extra-pair young across 32 species. The age of the sample at the time of observation was used as a covariate to control for fixation issues, and in all cases this was a nonsignificant covariate (all $t<1.35$, all $\mathrm{p}>0.14$ ). 95\% confidence intervals are presented $(\mathrm{LCL}$, lower confidence limit; UCL, upper confidence limit). Superscripts following $\lambda$ indicate significance levels of the likelihood-ratio tests against the model with maximum-likelihood value of $\lambda$ against $\lambda=1$ (first position) and $\lambda=$ 2 (second position). ${ }^{*} \mathrm{p}<0.05, * * * \mathrm{p}<0.001$

\begin{tabular}{|c|c|c|c|c|c|c|c|}
\hline Response & Predictor & df & $\mathbf{t}$ & $\mathbf{P}$ & Partial $r$ & LCL, UCL & $\lambda$ \\
\hline Percent abnormal sperm & Acrosome length & 33 & 2.227 & $0.033^{*}$ & 0.36 & $0.03,0.59$ & $0.744^{0.01,0.05}$ \\
\hline Percent abnormal sperm & Nucleus length & 33 & -3.505 & $0.001 *$ & -0.52 & $-0.70,-0.23$ & $0.403^{0.52,<0.01}$ \\
\hline Percent abnormal sperm & Membrane width & 33 & 6.064 & $<0.0001 * * *$ & 0.73 & $0.53,0.83$ & $0.000^{1,<0.01}$ \\
\hline Percent abnormal sperm & Head volume & 33 & 2.866 & $0.007^{*}$ & 0.45 & $0.13,0.65$ & $0.673^{0.06,0.09}$ \\
\hline Percent abnormal sperm & Waveform & 33 & 3.783 & $<0.001 * * *$ & 0.55 & $0.27,0.72$ & $0.521^{0.17,0.02}$ \\
\hline Percent abnormal sperm & Total sperm length & 33 & 2.469 & $0.019 *$ & 0.39 & $0.07,0.62$ & $0.734<0.01,0.04$ \\
\hline Percent abnormal sperm & Extra-pair young & 29 & -0.670 & 0.508 & -0.12 & $-0.44,0.24$ & $0.930<0.01,0.64$ \\
\hline
\end{tabular}


Table 2. PGLS model selection procedure. The percentage of sperm head abnormalities is the response variable in all models. Italics signify the variable which was removed in the subsequent model; bold signifies models within $2 \Delta$ AICc of the lowest AICc. ${ }^{*} p<0.05,{ }^{* *} p<0.01$.

\begin{tabular}{|c|c|c|c|}
\hline Model no & $\triangle \mathrm{AICC}$ & Akaike weight & Predictor variables \\
\hline Null model & 15.66 & 0.00 & 1 \\
\hline 1 & 7.99 & 0.01 & Acrosome length + Nucleus length + Membrane width + Head volume + Waveform $*+$ Total sperm length \\
\hline 2 & 4.93 & 0.04 & Acrosome length + Nucleus length + Membrane width + Waveform $*+$ Total sperm length \\
\hline 3 & 2.59 & 0.13 & Acrosome length + Membrane width* + Waveform** + Total sperm length \\
\hline 4 & 0.77 & 0.33 & Acrosome length + Membrane width** + Waveform** \\
\hline 5 & 0 & 0.49 & Membrane width** + Waveform** \\
\hline
\end{tabular}




\section{Supplementary material}

Supplementary file 1: Table S1 (Individual data points for each sperm cell and NHMO accession numbers), Table S3 (Species mean data from Støstad et al. (2018), including sperm traits based on SEM images as well as data on the rates of extra-pair young).

Supplementary file 2: Table S2 (PGLS analyses of sperm head abnormalities when analysed in separate categories), supplementary methods including Figure S1 (sperm head morphology measurements).

Supplementary file 3: Nexus file containing the phylogeny from Støstad et al. (2018). 

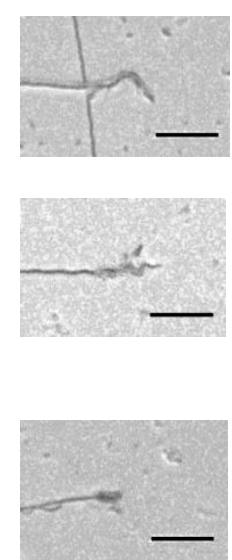


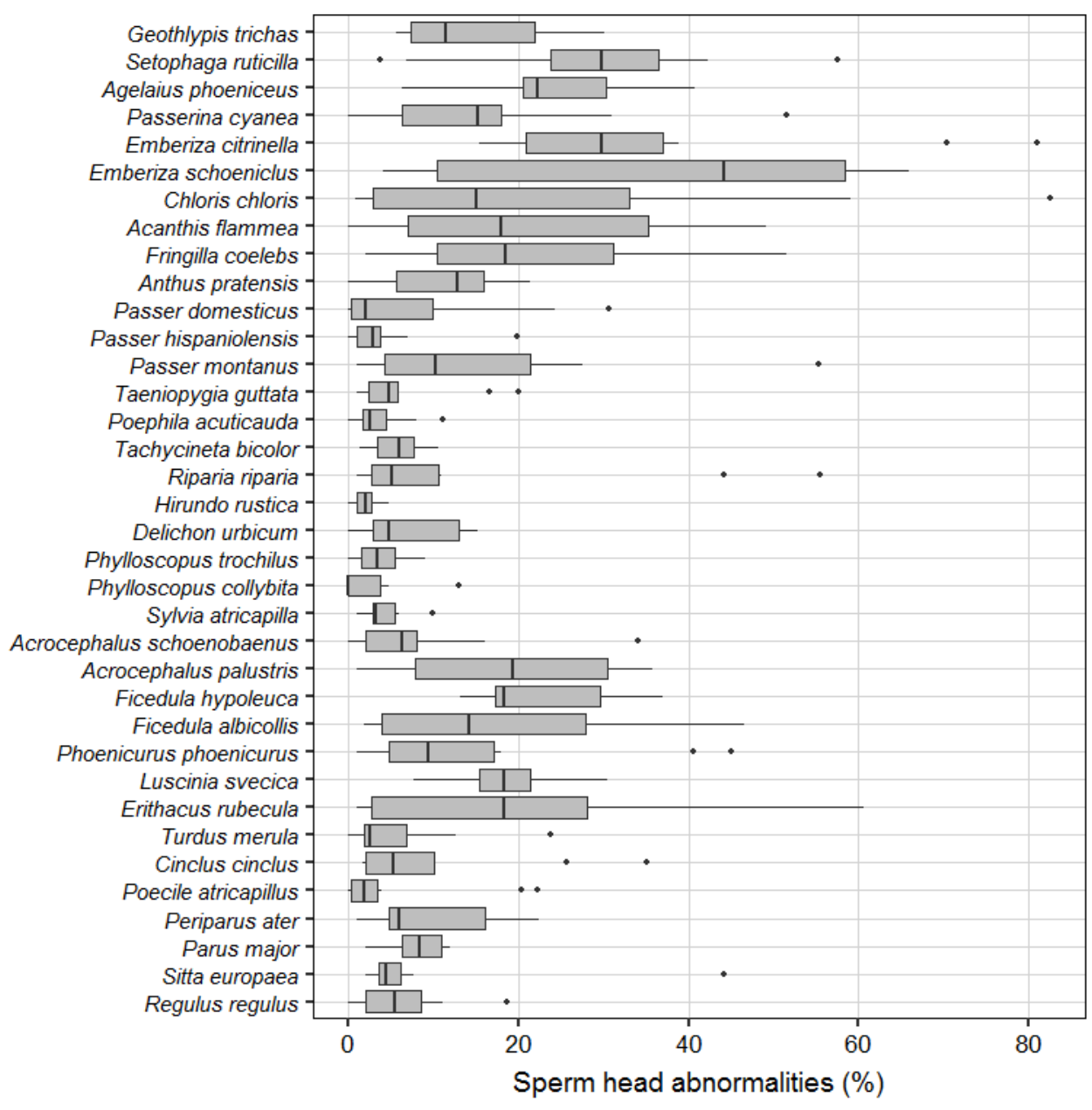



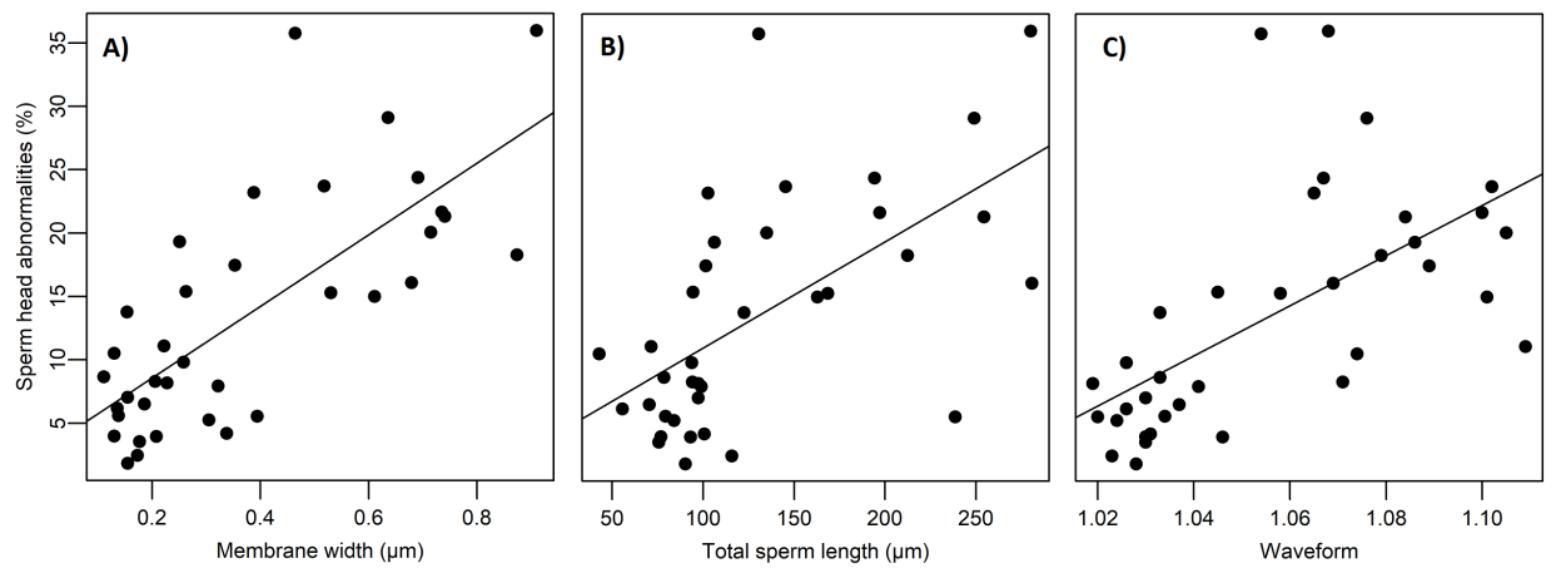

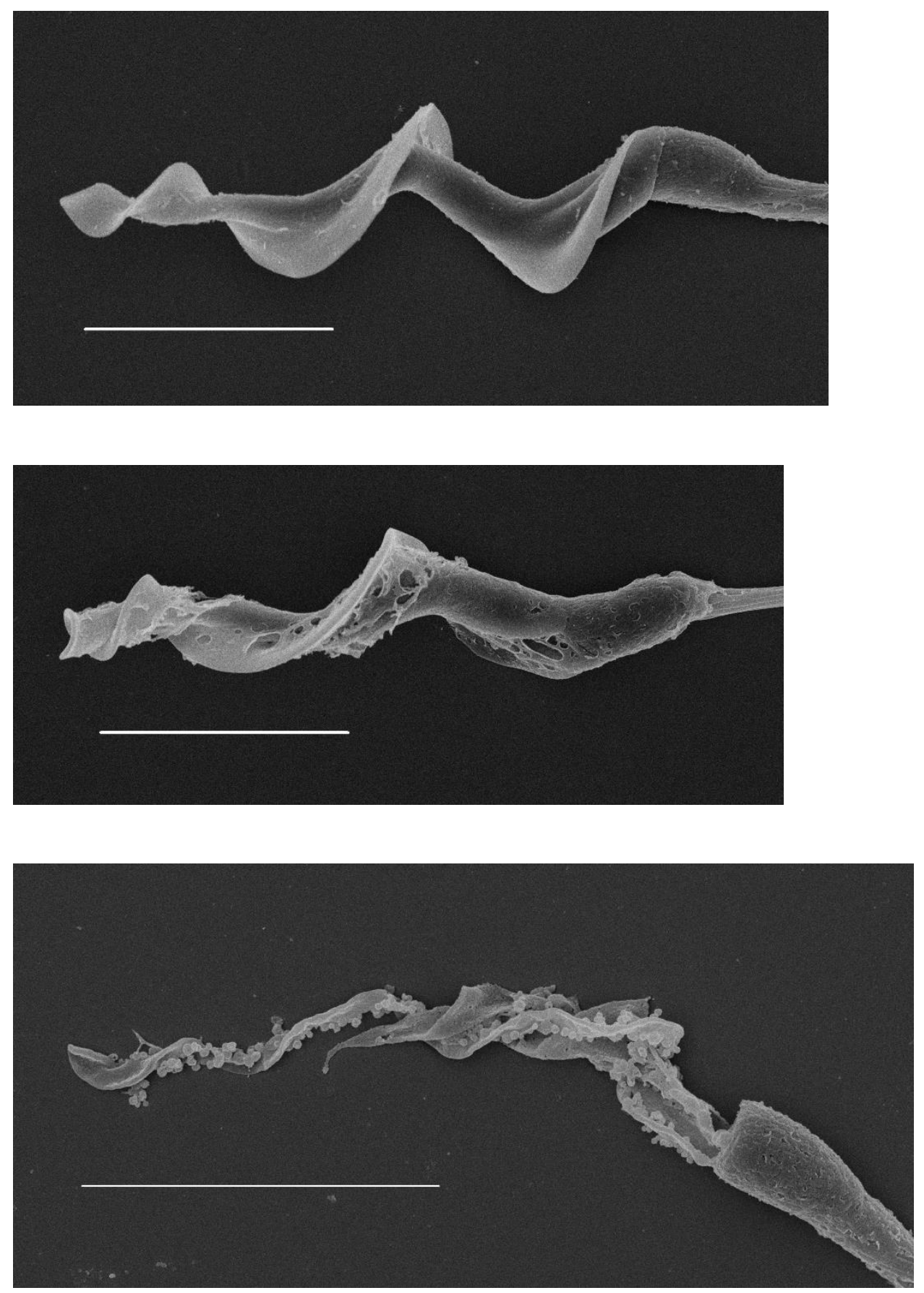